\title{
Neurogenic Bladder in Lyme Disease
}

\author{
Mi-hwa Kim, Won Chan Kim, Dong-Su Park ${ }^{1}$ \\ Departments of Neurology and ${ }^{1}$ Urology, CHA Bundang Medical Center, Seongnam, Korea
}

\begin{abstract}
Lyme disease is a multi-systemic, tick-borne infectious disease caused by a spirochete, Borrelia burgdorferi. Various urologic symptoms are associated with Lyme disease, which can be primary or late manifestations of the disease. Although voiding dysfunction is a rarely reported symptom in patients with Lyme disease, it is one of the most disabling complications of Lyme disease. Korea is not an endemic area of Lyme disease, thus, fewer cases have been reported. Herein, we report a case of a 32-yearold man with rapidly progressive bilateral ptosis, dysphagia, spastic paraparesis, and voiding difficulty in whom Lyme disease was diagnosed through serologic tests for antibodies and Western blot testing. A urodynamic study demonstrated detrusor areflexia and bulbocavernosus reflex tests showed delayed latency, indicating demyelination at S2-S4 levels. He received a 4-week course of intravenous ceftriaxone ( $2 \mathrm{~g} /$ day). The patient has recovered from the bilateral ptosis and spastic paraparesis but still suffers from neurogenic bladder.
\end{abstract}

Keywords: Lyme disease; Neurogenic urinary bladder; Urodynamics

Lyme disease is a systemic infection caused by a spirochete, Borrelia burgdorferi, which is mediated by ticks of the Ixodes ricinus complex that invades joints, nervous system, and cardiovascular system and triggers various manifestations, such as arthritis, encephalomyelitis, cranial neuropathy, polyneuritis, atrioventricular nodal block and myocarditis, along with the characteristic skin lesions, such as erythema migrans. Urinary dysfunction can also appear in Lyme disease. Korea is not an endemic region of Lyme disease. Therefore, prevalence of this disease is low in Korea and most previously reported cases are related to characteristic skin lesions. Herein, we report a rare case of Lyme disease accompanied by neurogenic bladder secondary to neurological involvement.

\section{CASE REPORT}

A 32-year-old male was hospitalized owing to bilateral ptosis, dysphagia, lower extremity weakness, and voiding difficulty. $\mathrm{He}$ was a Taekwondo master and his medical history was unremarkable. The patient informed that he had been admitted to an- other hospital due to an episode of sudden bilateral lower extremity weakness followed by bilateral ptosis and dysphasia two month prior to the admission. At that time, brain, cervical, and lumbar magnetic resonance imaging (MRI), electromyography (EMG), nerve conduction studies (NCS) and cerebrospinal fluid (CSF) examination were performed which revealed no abnormal findings. Clear causes for his condition were not identified and he was discharged after being instructed to manage severe urinary retention with clean intermittent catheterization. The bilateral ptosis and spastic paraparesis were somewhat relieved 2 weeks later, but the voiding dysfunction remained.

However, the symptoms were relapsed; bilateral ptosis was 2 weeks before; bilateral lower extremity weakness was 1 week before; dysphasia was 3 days before the admission. Neurological examinations revealed no other cranial nerve palsy apart from bilateral ptosis and dysphasgia. Spastic paraparesis was observed (dorsiflexion: Medical Research Council [MRC] grade 4, plantar flexion: MRC grade 4+) and both patellar and Achilles tendon reflexes were increased bilaterally. Babinskis sign and ankle clonus reflex were observed on both sides. Upper extrem-
Corresponding author: Won Chan Kim

Department of Neurology, CHA Bundang Medical Center, CHA University,

59 Yatap-ro, Bundang-gu, Seongnam 463-712, Korea

Tel: +82-31-780-5482 / Fax: +82-31-780-5269 / E-mail: wckim@cha.ac.kr

Submitted: September 7, 2012 / Accepted after revision: December 17, 2012
This is an Open Access article distributed under the terms of the Creative Commons Attribution Non-Commercial License (http://creativecommons.org/licenses/by-nc/3.0/) which permits unrestricted non-commercial use, distribution, and reproduction in any medium, provided the original work is properly cited. 
ity muscle strength, sensation and neurologic tests showed normal results. The patient's blood pressure, pulse rate, respiratory rate, and temperature were all normal. He complained of mild dyspnea during the admission and developed respiratory failure several days later which required endotracheal intubation.

Blood tests showed the patient's white blood cell concentration to be $7,760 / \mathrm{mL}$ (neutrophil, 63\%) and erythrocyte sedimentation rate to be $28 \mathrm{~mm} / \mathrm{hr}$. Serological test results for hepatitis B, human immunodeficiency virus and syphilis and autoantibody test results for cytomegalovirus, herpes simplex virus 1-2, toxoplasmosis, tumor markers (alpha-fetoprotein, careinoembryonal antigen, prostate-specific antigen, and carbohydrate antigen 19-9), paraneoplastic autoantibody and other autoantibodies were all negative. Cerebrospinal fluid (CSF) analysis revealed no evidence of inflammation with negative results for viruses, tuberculosis, and fungi and oligoclonal bands were absent. Brain and lumbar MRI was performed but no abnormal findings were found. EMG test results were normal; NCS showed decreased velocity in the bilateral superficial peroneal nerves, but there were no other abnormal findings. Acetylcholine receptor antibody was negative and there were no remarkable findings in repetitive nerve stimulation and neostigmine tests.

The patient was expectorating large amounts of sputum and third-generation cephalosporin (ceftriaxone $2 \mathrm{~g}$ /day) was administered to treat his pneumonia. The dyspnea and dysphagia were relieved 9 days after the treatment was commenced.

It was learned that the patient was positive for indirect immunofluorescence antibody (immunoglobulin $\mathrm{M}, 1: 16$; immunoglobulin G, 1:128) tests and Western blot tests for B. burgdorferi. These tests were repeated in 4 week time and confirmed positive results which suggested Lyme disease. He was treated with $2 \mathrm{~g}$ of intravenous ceftriaxone for 4 weeks and his condition was markedly improved although his voiding dysfunction remained without any improvement. A routine urine analysis showed no abnormal findings except for minor hematuria (urine red blood cells, 1-4/HPF), and there was no bacterial growth in a urine culture. When $300 \mathrm{~mL}$ of sterile distilled water was injected into the bladder for a urodynamic study, the patient felt micturition desire without an increase in intravesical pressure, suggesting detrusor areflexia (Fig. 1). Results of pudendal nerve sensory evoked potential tests and anal sphincter EMG, conducted 3 months after the admission, were normal; however, bulbocavernosus reflex tests showed delayed responses (right, $58.6 \mathrm{~ms}$; left, $68.2 \mathrm{~ms}$ ). Thus, demyelinating lesions at S2-S4 regions were suspected. Even after 4 months from the onset of

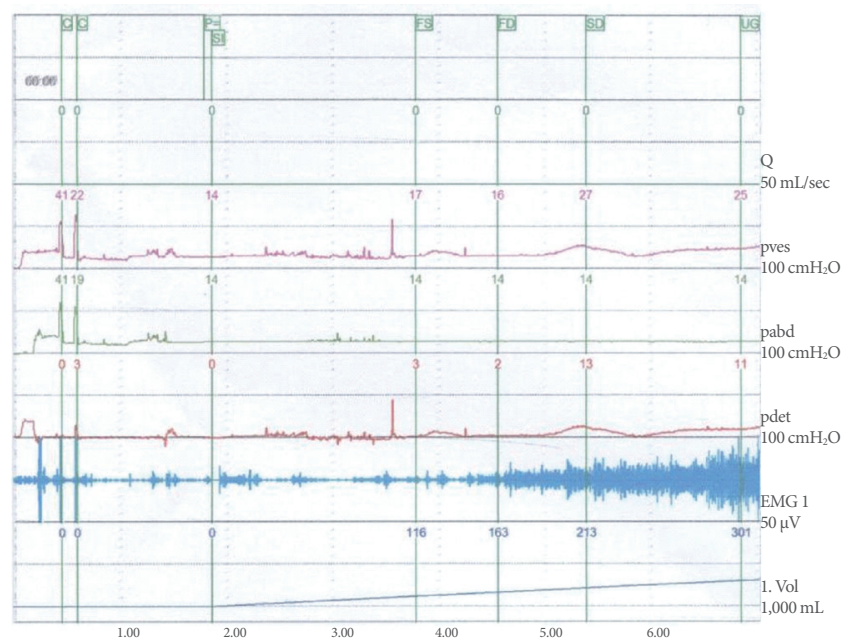

Fig. 1. The urodynamic study showed no voluntary or involuntary detrusor contraction on filling to $300 \mathrm{~mL}$. Pves, intravesical pressure; Pabd, abdominal pressure; Pdet, detrusor pressure; EMG, electromyography.

the symptom, the patient's voiding dysfunction has not been relieved and clean intermittent catheterization is still required to manage the problem.

\section{DISCUSSION}

Lyme disease is one of the most common tick-mediated infectious diseases in the United States and Europe. However, it is rarely reported in Korea. In Korea, a total of six cases of Lyme disease have been reported thus far. Except for two cases, one [1] in which non-typical skin lesions were shown and the other [2] in which musculoskeletal and cardiovascular invasions occurred, the remaining cases showed typical skin lesions. Neither neurological involvement nor persistent neurogenic bladder has been reported in patients with Lyme disease in Korea.

Lyme disease is largely divided into three stages. Early local infection stage is characterized by fever, headache and characteristic skin lesions called erythema migrans at the site of the tick bite. Disseminated infection stage is manifested by systemic symptoms involving cardiovascular, nervous, and musculoskeletal systems. Chronic atrophic acroangiodermatitis, chronic neurological symptoms, and arthritis are shown in chronic infection stage of the disease. However, these symptoms do not appear in order; above symptoms may simultaneously appear in multiple stages of the disease or symptoms suggesting chronic infection can appear without symptoms of the earlier stages [3]. The present patient showed neurological symptoms with- 
out symptoms of the initial stage of the infection, such as erythema migrans, fever, or headache; thus, it was though that this patient was in the disseminated or the chronic infection stage.

Lyme disease is diagnosed by clinical presentation and serological tests. Although it can be easily diagnosed if patients show characteristic skin lesions in endemic regions, the diagnosis may be delayed in regions where the disease does not occur frequently, such as Korea, or if patients show atypical symptoms. It is reported that approximately $31 \%$ of patients present with characteristic erythema migrans [4]. In such cases, serological tests play an important role in diagnosing the disease. The present patient visited this hospital after the acute stage and, thus, the characteristic skin findings could not be identified. Furthermore, no abnormality was found in imaging studies, and CSF analysis was also clear of infection. Although early diagnosis was difficult, Lyme disease was eventually diagnosed because the patient showed positive findings against B. burgdorferi in the serological tests along with rapidly progressing neurological symptoms. The patient had not visited any regions in which Lyme disease was endemic and had no memory of being bitten by a tick. Because ticks are very small in size, however, many patients will not realize that they have been bitten.

Among types of cranial nerve palsy shown in neuroborreliosis, facial palsy is the most frequent, and auditory nerve palsy is the second most frequent one [5]. The present patient showed bilateral oculomotor and glossopharyngeal nerve paralysis. Although lower cranial nerve paralysis is not a commonly reported symptom of Lyme disease, lower cranial nerve paralysis sometimes appears as the first symptom of neuroborreliosis $[5,6]$ when lower brain stem is involved and causes respiratory failure [7].

Voiding dysfunction can appear as an initial symptom or as a later-stage symptom of Lyme disease [8,9]. Micturition disorder generally develops after other neurological symptoms appear; however, it does sometimes appear in the early stage in a small number of cases [9].

Micturition disorders in Lyme disease can occur by several mechanisms. First, Lyme cystitis can occur because the spirochete directly invades the urinary bladder [9]. The second mechanism is related to neuroborreliosis, such as meningoencephalopathy, transverse myelitis, myeloradiculitis, and demyelinating lesions of the spinal cord. Micturition disorders can appear in diverse forms such as detrusor hyperreflexia, detrusor-sphincter dyssynergia, and detrusor areflexia [8]. A study reported that 11 of 44 Lyme encephalomyelitis patients showed urinary bladder dysfunction [10] and another study reported that 5 out of 7
Lyme encephalomyelitis patients showed detrusor hyperreflexia and the remaining 2 patients showed detrusor areflexia [9]. The present patient showed detrusor areflexia in a urodynamic study.

The neurological symptoms caused by Lyme disease are mostly relieved when patients are treated with antibiotics within an appropriate time period. However, in this case, his voiding dysfunction was not relieved while other neurological symptoms were relieved by a 4 -week antibiotic treatment. Judging based on bulbocarvernosus reflex tests and suspected demyelinating lesions in S2-S4 regions, it was assumed that his neurogenic bladder was caused by neuroborreliosis although the patient's urinary bladder tissues were not examined.

Although Korea is not a region where Lyme disease frequently occurs, it is necessary to be well-acquainted with the neurological symptoms that can appear in Lyme disease to facilitate early diagnosis of Lyme disease in patients with rapidly developing neurological symptoms, including voiding dysfunction so that they can receive appropriate treatment.

\section{CONFLICT OF INTEREST}

No potential conflict of interest relevant to this article was reported.

\section{REFERENCES}

1. Kim JW, Kim JS. A case of Lyme disease with unusual cutaneous manifestations. Korean J Dermatol 2005;43:501-6.

2. Lee D, Kim SH, Hong SK, Seo JK, Sung HS, Hwang SW. A case of Lyme disease with various general symptoms. Korean J Dermatol 2008;46:1112-6.

3. Kim TH, Choi EH, Lee MG, Ahn SK. Serologically diagnosed Lyme disease manifesting erythema migrans in Korea. J Korean Med Sci 1999;14:85-8.

4. Kohlhepp W, Kuhn W, Kruger H. Extrapyramidal features in central Lyme borreliosis. Eur Neurol 1989;29:150-5.

5. Gustaw K, Mirecka U. Dysarthria as the isolated clinical symptom of borreliosis: a case report. Ann Agric Environ Med 2001;8:95-7.

6. Velazquez JM, Montero RG, Garrido JA, Tejerina AA. Lower cranial nerve involvement as the initial manifestation of Lyme borreliosis. Neurologia 1999;14:36-7.

7. Silva MT, Sophar M, Howard RS, Spencer GT. Neuroborreliosis as a cause of respiratory failure. J Neurol 1995;242:604-7.

8. Chancellor MB, Dato VM, Yang JY. Lyme disease presenting as urinary retention. J Urol 1990;143:1223-4. 


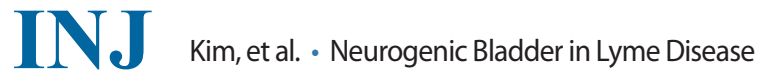

9. Chancellor MB, McGinnis DE, Shenot PJ, Kiilholma P, Hirsch IH. Urinary dysfunction in Lyme disease. J Urol 1993;149:26-30.

10. Ackermann R, Rehse-Kupper B, Gollmer E, Schmidt R. Chronic neurologic manifestations of erythema migrans borreliosis. Ann N Y Acad Sci 1988;539:16-23. 